\title{
Improvement of Tribological Properties of Epoxy Resin by Addition of Oxidized Nanocarbons
}

\author{
Naohiro Matsumoto ${ }^{1)^{*}}$, Yusuke Yukiyoshi ${ }^{2)}$, Yuya Omiya $^{2)}$ and Hiroshi Kinoshita ${ }^{1)}$ \\ ${ }^{1)}$ Department of Mechanical Engineering, Graduate School of Engineering, University of Hyogo, \\ 2167 Shosya, Himeji, Hyogo 671-2280, Japan \\ ${ }^{2)}$ Graduate School of Natural Science and Technology, Okayama University, \\ 3-1-1 Tsushima-naka, Kita-ku, Okayama 700-8530, Japan \\ *Corresponding author: Naohiro Matsumoto (matsumoto@eng.u-hyogo.ac.jp)
}

Manuscript received 30 September 2019; accepted 17 March 2020; published 30 April 2020

Presented at the International Tribology Conference Sendai 2019, 17-21 September, 2019

\begin{abstract}
Graphene oxide (GO) and oxidized wood-derived nanocarbon (oWNC) were dispersed in an epoxy resin to improve the mechanical and tribological properties of an epoxy resin. Mechanical properties of the GO/epoxy (GO/EP) and oWNC/epoxy composites (oWNC/ EP) were investigated by tensile tests and nano-indentation. Under dry, water, and oil lubrication conditions, friction coefficients and wear volumes of these composites were measured. GO has single-layered structure. oWNC has chain-like nanostructures, and the nanochains are aggregated. GO and oWNCs have same surface chemical composition due to the same oxidation treatment. Thus, in this study, the structure and morphology dependences of the nanocarbons in mechanical and tribological properties will be appeared when the nanocarbons were used as the additives in the resin. The tensile strength, tensile modulus, and hardness increased by $20 \%$, $63 \%$, and $35 \%$ in maximum by the addition of GO and oWNC to the epoxy resin, respectively. Moreover, the wear resistance of GO/EP and oWNC/EP was enhanced compared to that of a neat epoxy. The reduction rates of the wear volume for $5.0 \mathrm{mass} \% \mathrm{GO} / \mathrm{EP}$ and 1.0 mass $\%$ oWNC/EP achieved to $91 \%$ and $67 \%$, respectively. Comparing GO and oWNC, oWNC effectively reduced the friction coefficient and wear at a lower concentration. The improvement of tribological properties after the addition of the oxidized nanocarbons can be attributed to the increase in the hardness and lubricity of the composites.
\end{abstract}

\section{Keywords}

oxidized nanocarbon, graphene oxide, epoxy resin, friction, wear resistance

\section{Introduction}

Resin-based composites can improve energy efficiency in transport machinery owing to their high strength and low weight. However, tribological properties of these composites should be improved prior to usage in mechanically sliding elements. Because the tribological properties of resin-based composites are insufficient to particularly achieve the desired and better wear durability of mechanical sliding than that obtained by metal components, attempts have been undertaken to improve their properties by adding nanomaterials in resins [1-6]. Epoxy-based resin is widely used as a matrix for fiber reinforced plastic. The wear properties of epoxy resins are reportedly improved by the addition of Si-based [1-3] or metal oxide nanoparticles [4-6]. Graphitic nanomaterials such as carbon nanotube and graphene are known additives to improve the tribological properties of resins because they have high solid lubricity and chemical inertness [7-19]. Carbon nanotube improved the wear resistance of epoxy resin with the amount of $0.1-1.0$ mass $\%[7,8,15,17]$. Graphene oxide (GO), which has a two-dimensional sheet-like structure, showed an improvement in friction, wear resistance, and mechanical properties of epoxy resin $[9,12,16-20]$. It was assumed that the strong interaction between GO and epoxy might affect the mechanical and tribological properties. Nanocarbon materials were highly expected to improve the tribological properties of epoxy resin although their ideal configurations were less understood.

Oxidized wood-derived nanocarbons (oWNC) mainly consist of spherical graphitic structures with nano-sizes and, similar to GO, have many oxygen functional groups on the surfaces $[21,22]$. It is therefore possible that the interaction strength between epoxy resin and oWNC mimics that of GO. These materials can be as the additives to improve the base materials properties. In this study, GO and oWNC 
were dispersed in an epoxy resin to investigate the effect on mechanical and tribological properties of the GO/epoxy (GO/ $\mathrm{EP}$ ) and oWNC/epoxy composites (oWNC/EP) under dry, water, and oil lubrication conditions. The mechanical properties of tensile strength and tensile modulus were evaluated using the tensile tests, while the indentation hardness was evaluated using the nano-indenter. Reciprocation tribology tests were conducted under dry, water, and oil lubrication conditions. The effect of different nanocarbons of sheet-like GO and spherical oWNC on the enhancement of mechanical and tribological properties under various lubrication conditions was discussed.

\section{Experiment}

\subsection{Nanocarbon materials}

Detailed synthesis methods of GO and oWNCs were explained in a previous study [21-24]. A single-layered GO with epoxy, hydroxyl, and carboxyl functional groups on the surface was obtained by oxidizing and peeling graphite by the modified Hummer's method. oWNC was prepared from wood powders by metal-catalyzed carbonization. Wood particles were mixed with the aqueous solution of iron nitrate and heated at $850^{\circ} \mathrm{C}$ in a nitrogen atmosphere for $1 \mathrm{~h}$. After purification, wood-derived nanocarbon (WNC), which has a graphitic structure, was obtained. oWNC was also obtained via the oxidation treatment using the modified Hummer's method, which introduced the oxygen functional groups to the surface. oWNCs have similar surface chemical composition as GO due to the same oxidation treatment. Figure 1 shows representative scanning electron microscopy (SEM) images of the GO and oWNCs. GO has the size of about $10 \mu \mathrm{m}$ with the thickness of $1 \mathrm{~nm}$. oWNC has chain-like aggregated nanostructures. The primary particle size of oWNC is about $20 \mathrm{~nm}$.

\subsection{Composite specimens}

Bisphenol-A epoxy resin (JER828 with ST11 hardener, Mitsubishi Chemical Corporation) was used as a base material. GO and oWNC were dispersed in epoxy resin in various concentrations (0.5-5.0 mass\%) using ultrasonication. After defoaming in vacuum, epoxy resin was cured for $24 \mathrm{~h}$ at room temperature and for $2 \mathrm{~h}$ at $80^{\circ} \mathrm{C}$ with the thickness of $0.1 \mathrm{~mm}$. Figure 2 shows the optical microscope images of the specimens with the addition of 0.5 and 1.0 mass $\%$ of each GO and oWNC. Dispersed particles in the epoxy resin were observed. Comparing 1.0 mass\% of GO/EP and oWNC/EP in Fig. 2 (c) and (e), agglomerated features were observed for oWNC/EP. The surface morphologies of the composite specimens were observed by the atomic force microscopy (AFM), as shown in Fig. 3. As observed, the surfaces were roughened by the addition of the GO and oWNC. Figure 4 shows the surface roughness $(\mathrm{Ra})$ of the specimens measured by AFM in $10 \mu \mathrm{m}$ as a function of additive concentration. Ra has increased in the range of $0.05-0.10 \mu \mathrm{m}$ with increasing additive concentration for both GO/EP and oWNC/EP.

\subsection{Mechanical properties measurements}

The mechanical properties of specimens were measured by tensile and nanoindentation tests. For tensile tests, dumbbellshaped specimens were prepared by mechanically cutting the cured epoxy resin using mold (length of $35 \mathrm{~mm}$ and width of 6 $\mathrm{mm}$ and the length of narrow part as $12 \mathrm{~mm}$ with $2 \mathrm{~mm}$ width). Figure 5 shows the photographs of the specimens with different concentrations of GO and oWNCs. The color of the specimens has changed to black with increasing concentration of GO and oWNC. When the concentration of GO was increased to 5.0 mass $\%$, the specimen cracked. Tensile tests were conducted at $0-3.0$ mass\% for GO/EP and 0-5.0 mass\% for oWNC/EP to measure the tensile strength and tensile modulus averaged by 5 specimens. Indentation hardness was measured by the nanoindentation test at the maximum indentation load of 100 $\mathrm{mN}$. The measurements were carried out 100 times using the Berkovich tip for each specimen with the interval length of 75 $\mu \mathrm{m}$ to evaluate the averaged indentation hardness.

\subsection{Tribology measurements}

Tribological properties of the composite specimens were measured using the reciprocating pin-on-plate friction tests under dry, water, and oil lubrication conditions. Distilled water and polyalphaolefin (PAO4) were used as lubricants. Epoxy plates containing GO or oWNC were tested by using JIS-SUJ2 balls with the diameter of $2 \mathrm{~mm}$ as the sliding counterpart. The normal load of $1 \mathrm{~N}$ for dry and water lubrication and 1 or $3 \mathrm{~N}$ for oil lubrication were applied by dead weight. The reciprocation amplitude of $2 \mathrm{~mm}$ and the frequency of $5 \mathrm{~Hz}$ were applied for 20,000 or 40,000 cycles. Friction forces were measured by the strain gauge installed on the ball side. Wear volumes were evaluated by the cross-sectional area of the wear trac, which is measured by a laser microscopy, multiplied by the length of wear trac. Specific wear rates were calculated by the wear volume at unit sliding length and load.

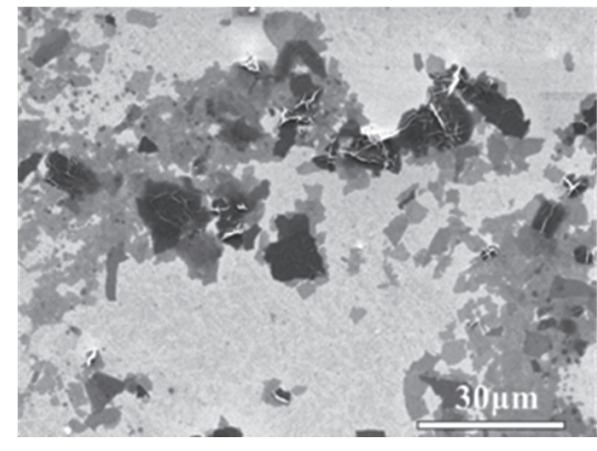

(a) Graphene Oxide

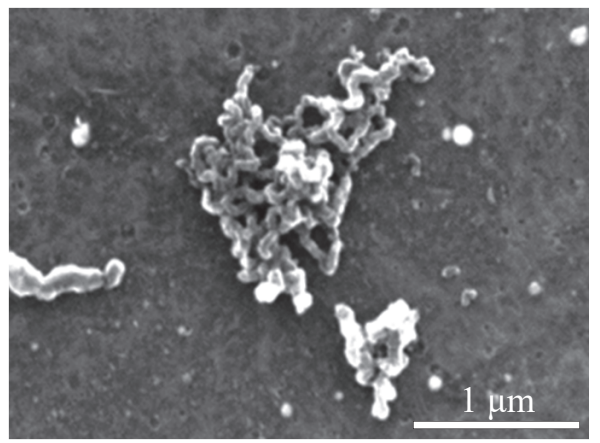

(b) Oxidized Wood-derived Nano Carbon

Fig. 1 Representative SEM images of (a) GO and (b) oWNCs 


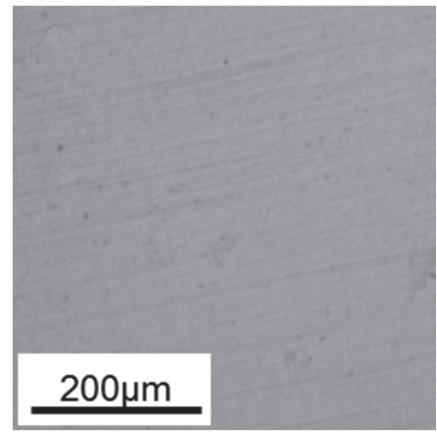

(a) Neat EP

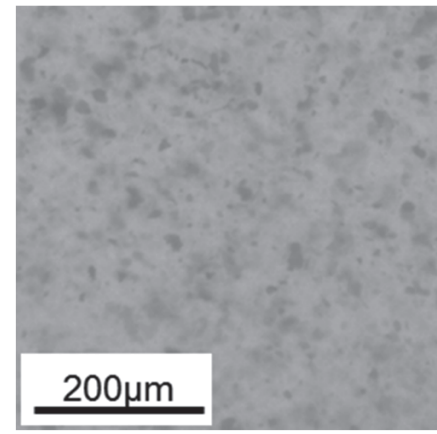

(b) 0.5 mass $\%$ GO/EP

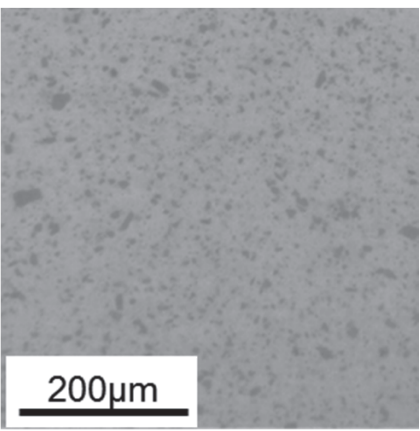

(d) 0.5 mass \% oWNC/EP

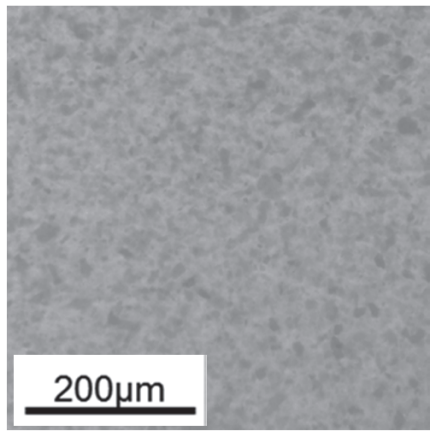

(c) 1.0 mass \% GO/EP

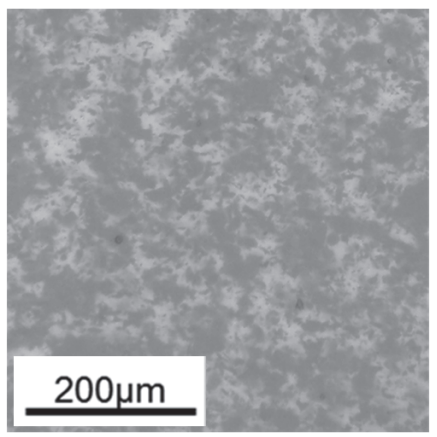

(e) 1.0 mass $\%$ oWNC/EP

Fig. 2 Optical microscope images of (a) neat epoxy, (b) 0.5 mass\% GO/EP, (c) 1.0 mass $\%$ GO/EP, (d) 0.5 mass\% oWNC/EP, (e) 1.0 mass $\%$ oWNC/EP
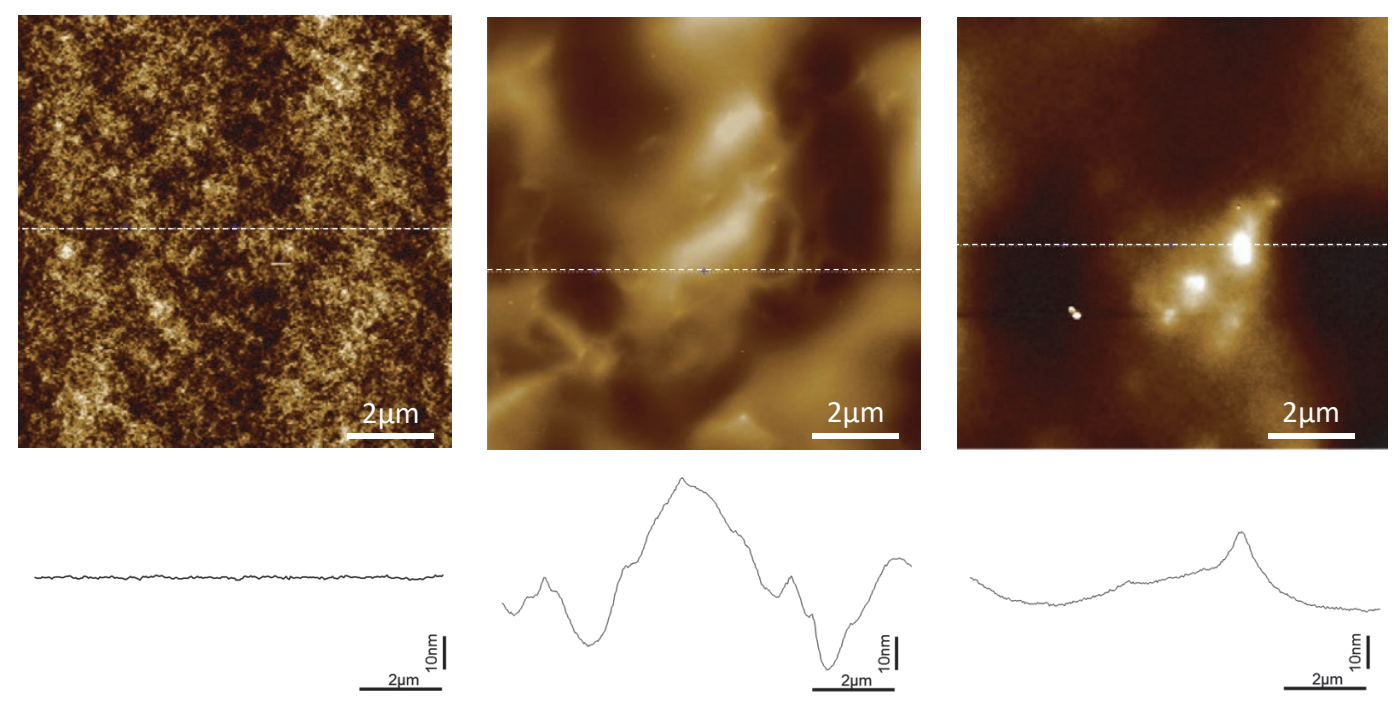

(a) Neat EP

(b) 1 mass $\%$ GO/EP

(c) 1 mass \% oWNC/EP

Fig. 3 AFM images of (a) neat EP, (b) 1.0 mass $\%$ GO/EP, and (c) 1.0 mass $\%$ oWNC/EP

\section{Results}

3.1 Mechanical properties of epoxy resin by addition of nanocarbons

Figure 6 shows the tensile test results for the tensile strength and tensile modulus of GO/EP and oWNC/EP as a function of the additive concentration. The additive concentration at 0 mass $\%$ corresponds with the neat epoxy specimens. The tensile strength of GO/EP increased with the concentration until 0.5 mass $\%$ and decreased until 3.0 mass $\%$ compared with that of the neat epoxy. Similarly, the tensile strength of the oWNC/EP increased until 3.0 mass $\%$ and decreased at 5.0 mass $\%$, which is a little higher than that of GO/EP. The tensile modulus of $\mathrm{GO} / \mathrm{EP}$ also increased with the increasing concentration until 1.0 mass $\%$ and remained stable. On the contrary, the tensile modulus of the oWNC increased almost monotonically with 
the concentration until 5.0 mass $\%$. The degradation of the tensile strength for GO/EP and oWNC/EP at relatively high concentration is considered that the stress concentration at the fillers under the increased elastic modulus caused the breaking of the resin with lower tensile load.

Figure 7 shows the indentation hardness as a function of the nanocarbon concentration. The indentation hardness of the $\mathrm{GO} / \mathrm{EP}$ and oWNC/EP increased lineally with the increasing concentration. However, the increasing effect was slightly higher for GO/EP than that for the oWNC/EP. Considering the almost linear increase of the hardness with the increased concentration which measured in compressive direction, interfacial debonding of the fillers can be occurred in the tensile direction.

\subsection{Tribological properties under dry sliding condition}

Figure 8 shows friction measurement results of the neat epoxy and 1.0 mass $\%$ GO/EP under the dry sliding condition. The friction coefficient of neat epoxy started at about 0.5 and remained almost constant until 20,000 cycles. As for 1.0 mass\% $\mathrm{GO} / \mathrm{EP}$, although the friction coefficient started below 0.2 , it has

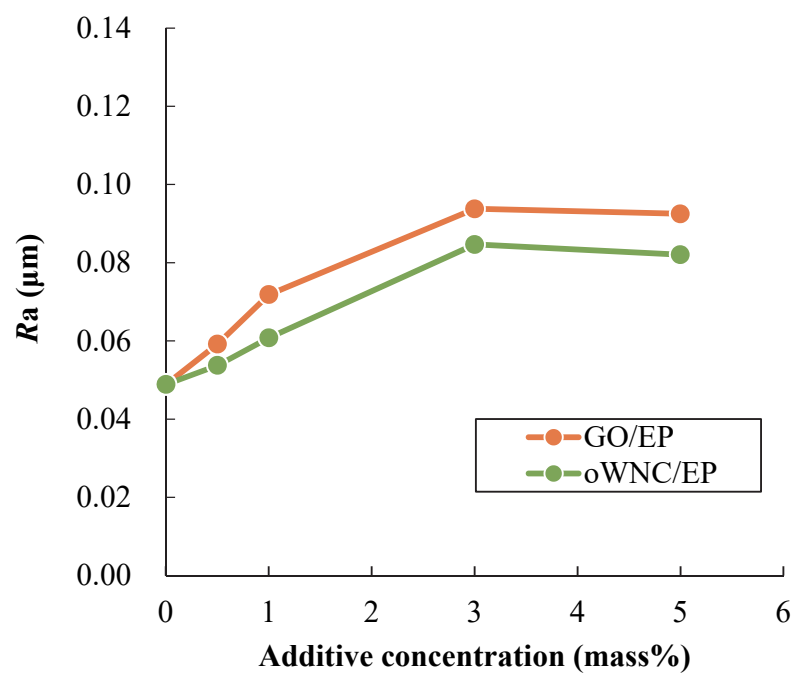

Fig. 4 Surface roughness (Ra) of GO/EP and oWNC/EP specimens as a function of additive concentration

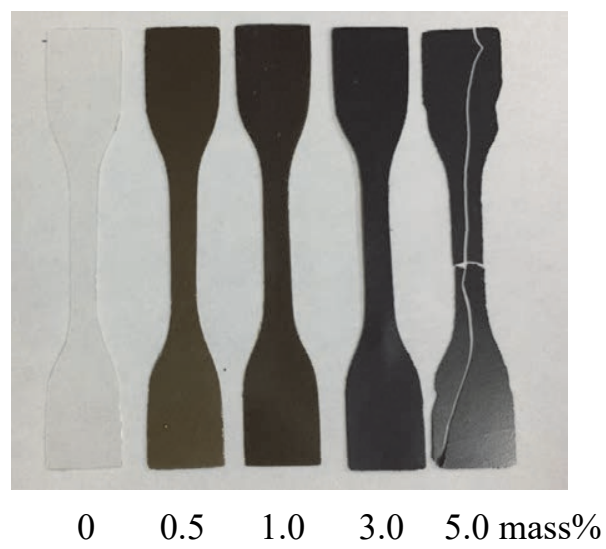

(a) GO/EP quickly increased to about 0.5 , thus equaling the stable value of the neat epoxy. Figure 9 shows the optical microscope images of the plate surface after dry sliding. The wear tracks were clearly observed for both neat epoxy and GO/EP. In the case of neat epoxy, plowings of the resin surface were observed and the left side of the wear track was upheaved. In contrast, the surface of epoxy with GO was relatively smoother and the wear was

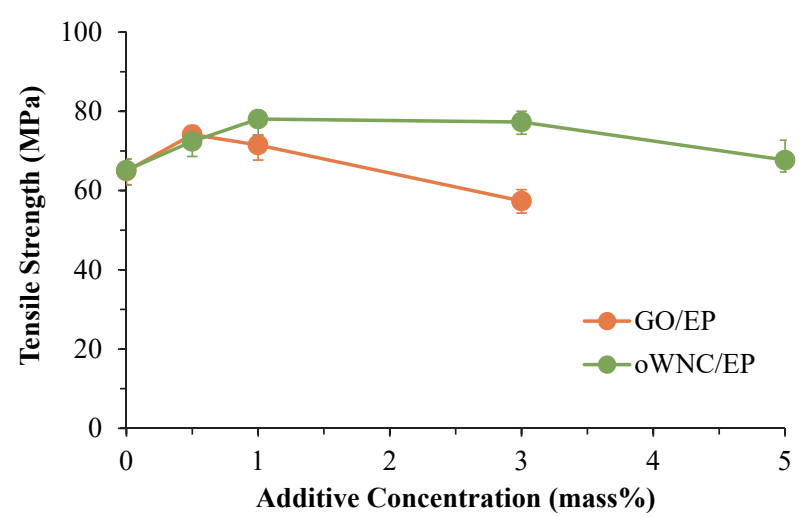

(a) Tensile Strength

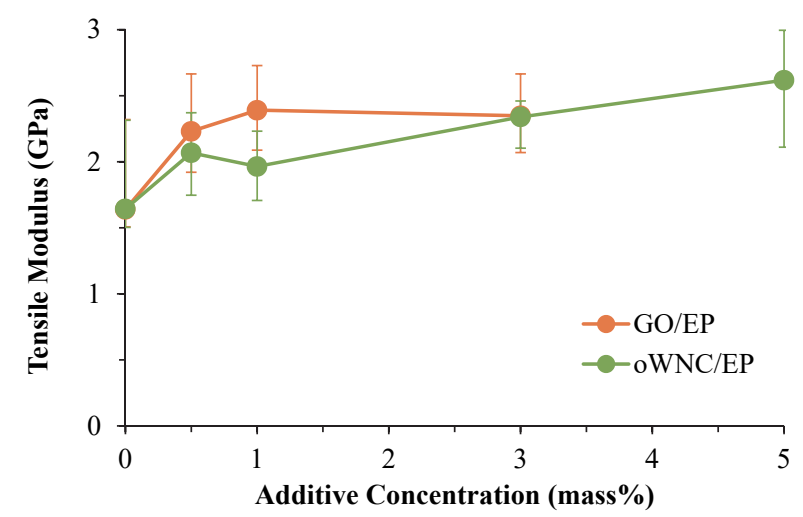

(b) Tensile Modulus

Fig. 6 Mechanical properties of (a) tensile strength and (b) tensile modulus of GO/EP and oWNC/EP with increasing additive concentrations

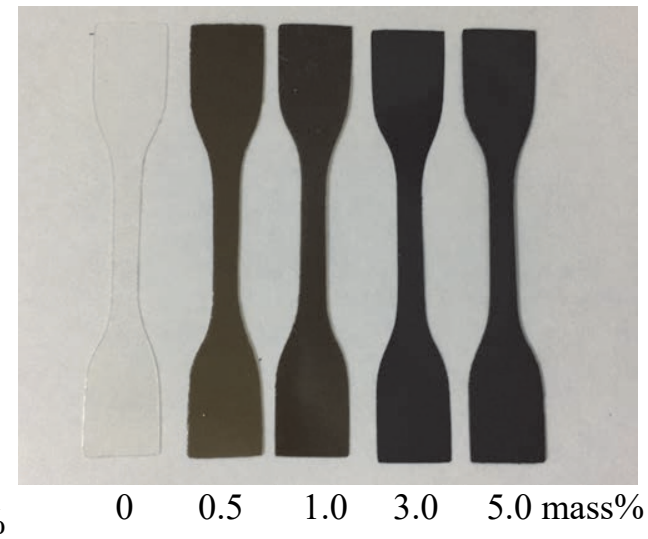

(b) oWNC/EP

Fig. 5 Photographs of the composite specimens of (a) GO/EP and (b) oWNC/EP for tensile test at various additive concentrations at $0-5.0$ mass $\%$ 


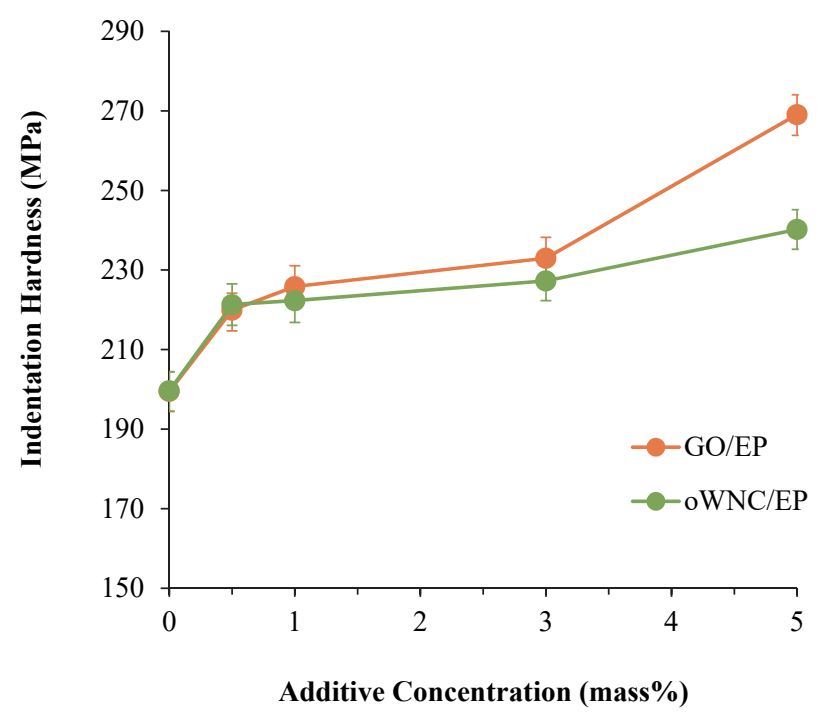

Fig. 7 Indentation hardness of GO/EP and oWNC/EP with increasing additive concentration

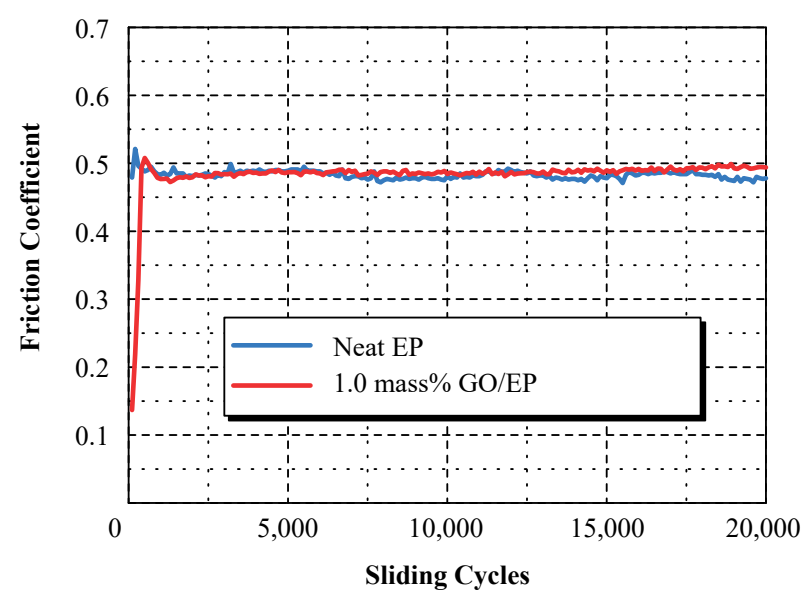

Fig. 8 The transition of friction coefficient of GO/EP under dry sliding condition

lesser than those of the neat epoxy. Therefore, the addition of 1.0 mass\% of GO in epoxy improved anti-wear properties under a dry sliding condition.
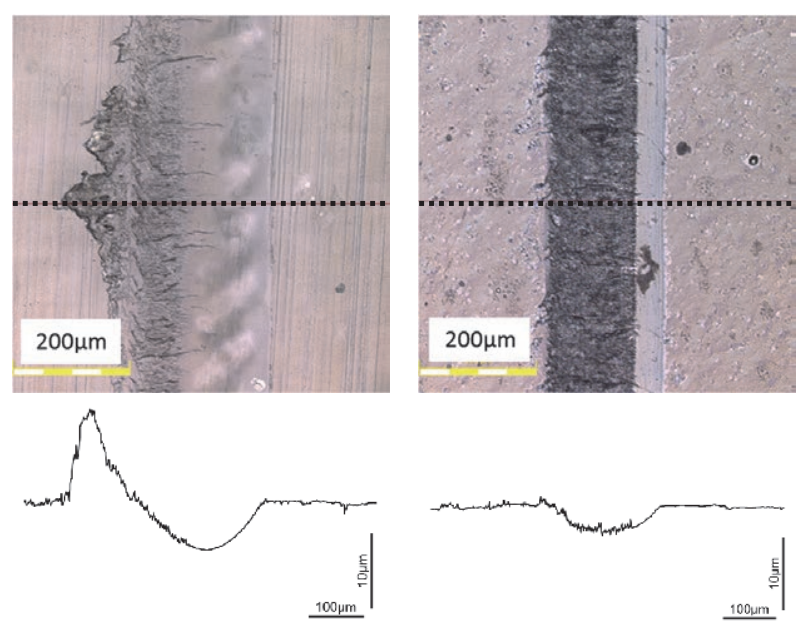

(a) Neat EP

(b) 1.0 mass $\%$ GO/EP

Fig. 9 Optical microscope images of the wear track of (a) neat epoxy and (b) 1.0 mass\% GO/EP after dry sliding

3.3 Under water-lubricated sliding condition

Figure 10 shows the friction coefficients of 0-5.0 mass \% GO/ EP under a water lubrication condition. The friction coefficient of neat epoxy under water lubricated condition was almost same level as that of the dry sliding condition. By increasing the content of GO in epoxy, the initial friction coefficients were drastically reduced below 0.1 gradually increased after increasing the sliding cycles. At the stabled friction coefficient at around 40,000 cycles, lower friction coefficient was kept for the GO/EP with the GO content more than 3.0 mass\%. Larger amount of GO shows lower friction coefficient because more GO can be supplied in the sliding interface to reduce friction. Additionally, the decrease of the wear amount of the resin (show later) may affect the lowering friction coefficient. Figure 11 shows the optical microscope images of the sliding surfaces of GO/EP under water lubrication. The wear track of the neat epoxy under water lubrication was significant compared to that of the dry sliding surface. The plowing of the resin surface was not observed. With the addition of GO in epoxy, the wear was gradually reduced by increasing the concentration of the GO. Figure 12 shows the friction coefficients of $0-5.0$ mass $\%$ oWNC/EP under the water lubrication condition. Significant

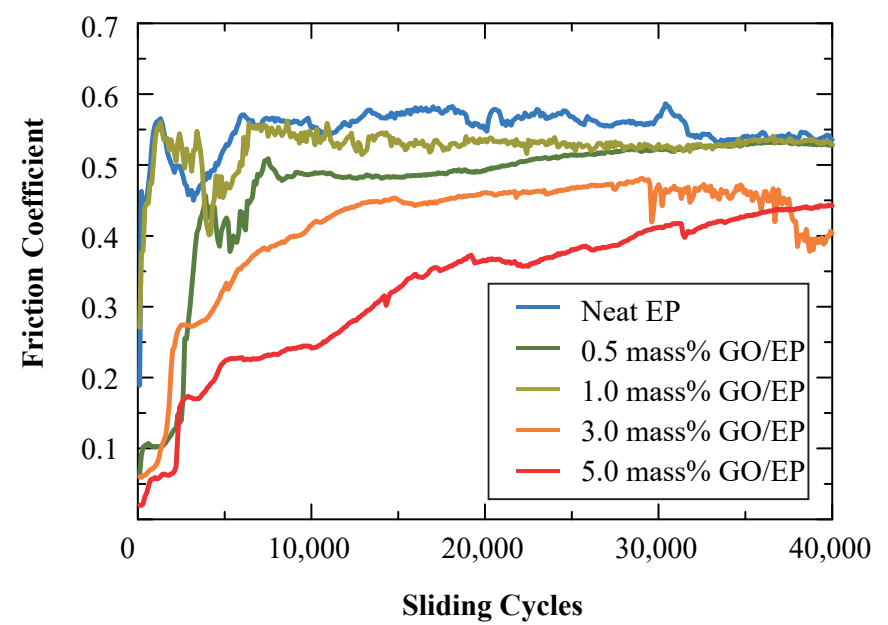

Fig. 10 Friction coefficient of 0-5.0 mass $\%$ GO/EP under water lubrication condition 

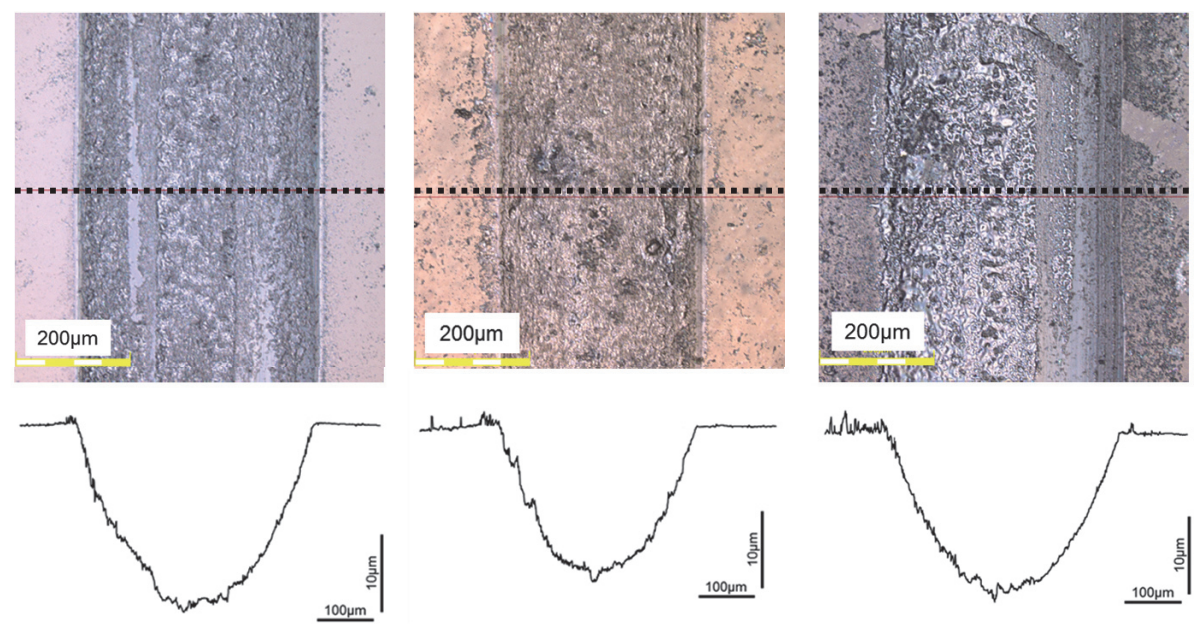

(a) Neat EP

(b) 0.5 mass $\%$ GO/EP

(c) 1.0 mass $\%$ GO/EP
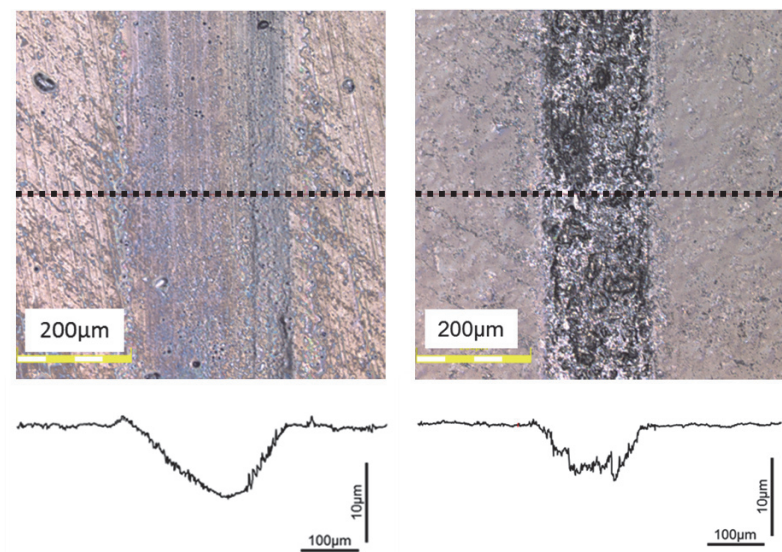

(d) 3.0 mass $\%$ GO/EP

(e) 5.0 mass $\%$ GO/EP

Fig. 11 Optical microscope images of the sliding surface of (a) neat epoxy, (b) 0.5 mass\%, (c) 1.0 mass $\%$, (d) 3.0 mass $\%$, and (e) 5.0 mass $\%$ GO/EP under water lubrication

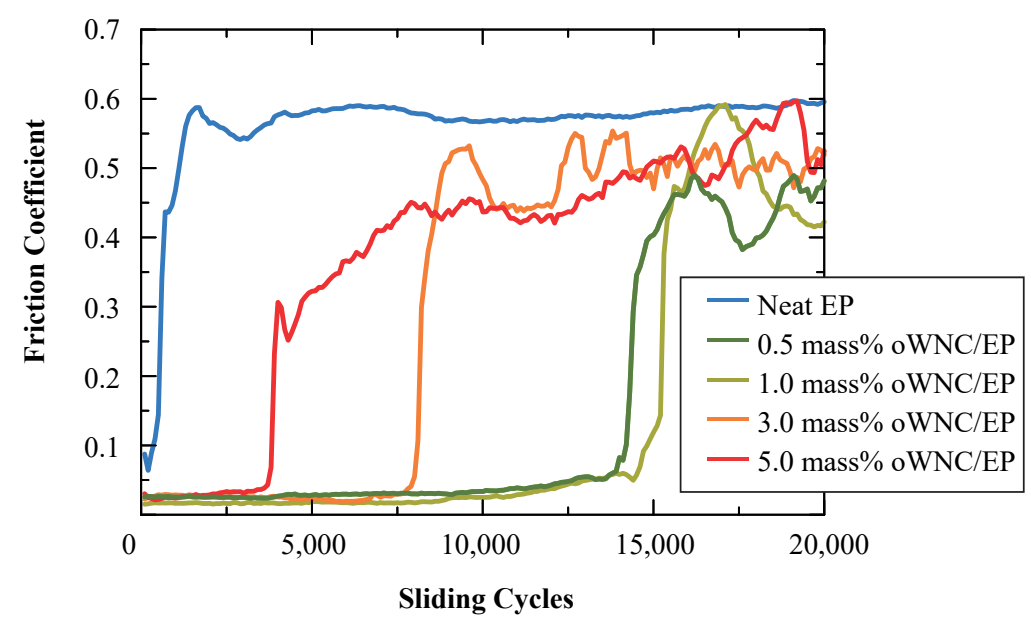

Fig. 12 Friction coefficients of 0-5.0 mass $\%$ oWNC/EP under water lubrication condition 
reductions of the friction coefficients were observed for oWNC/ EP. The starting levels of friction coefficients were below 0.05 for all the specimens containing oWNC at more than 0.5 mass $\%$. By increasing the friction cycles, the friction coefficients were rapidly increased and then remained stable. The duration of the initial low friction state was maintained for a longer time with smaller concentrations of oWNC at 0.5 or 1.0 mass $\%$ compared to that with higher concentrations at 3.0 or 5.0 mass $\%$. Figure 13 shows the optical microscope images of the sliding surfaces of oWNC/EP under the water lubrication. The trend of wear size with the additive concentration for oWNC/EP was different from GO/EP. The wear is less at the lower concentrations of 0.5 and 1.0 mass $\%$ than that at the higher concentrations of 3.0 and 5.0 mass\%. The tradition of friction coefficient of oWNC/ EP under the water lubrication condition can be related to the wear of resin. The rise of friction coefficient was relatively quick for the 3.0 and 5.0 mass $\%$ oWNC/EP, which were high wear amount. One of the possible reasons is that increased contact area between ball and resin with progressed wear caused the increase of the friction coefficient.

Figure 14 shows the wear scars of the counterpart specimen of SUJ2 ball. It was observed that the circle-shaped wear scar was at the center and black deposits were adhered to it for the ball surface sliding with neat epoxy, 1.0 mass $\%$ GO/EP, and 0.5 mass $\%$ oWNC/EP plates. The diameters of the wear scars on the SUJ2 balls were smaller than the width of wear tracks of epoxy plates. This means the wear was significant for the epoxy plate surface. Comparing the wear scars of the balls slide against different epoxy plates, the diameter of wear scars for 0.5 mass $\%$ oWNC/EP was relatively large.

\subsection{Under oil-lubricated sliding condition}

Figure 15 shows friction coefficients of $0-5.0$ mass $\%$ of GO/ EP under an oil lubrication condition. PAO4 was used as the lubrication oil. The friction coefficient of neat epoxy was in the range of $0.05-0.15$, which was lower than that under the dry and water lubrication conditions. The sliding was under boundary lubrication condition since the wear track was clearly observed for the plate surface. GO/EP specimens with the concentration of $0.5-5.0$ mass $\%$ indicated friction reduction by about half. The transition of the friction coefficients of the GO/ EP was relatively stable at $0.02-0.06$ compared to that under the dry and water lubrication conditions. The friction coefficient is approximately the same as the one at more than 0.5 mass\%, which is independent of the GO concentration. Figure 16 shows the optical microscope images of the sliding surfaces of GO/ EP under the water lubrication. For the neat epoxy, the wear track was clearly observed under boundary lubrication. On the contrary, a very small wear track was observed for GO/EP at the concentration of more than 0.5 mass $\%$. The friction coefficients of $0-5.0$ mass $\%$ oWNC/EP under oil lubrication conditions are shown in Fig. 17. The friction coefficients of the oWNC/EP at the concentration of more than 0.5 mass $\%$ started at about 0.03 and gradually increased like GO/EP under the oil lubrication

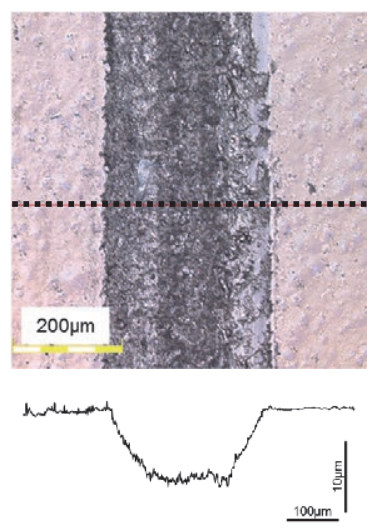

(a) 0.5 mass \% oWNC/EP
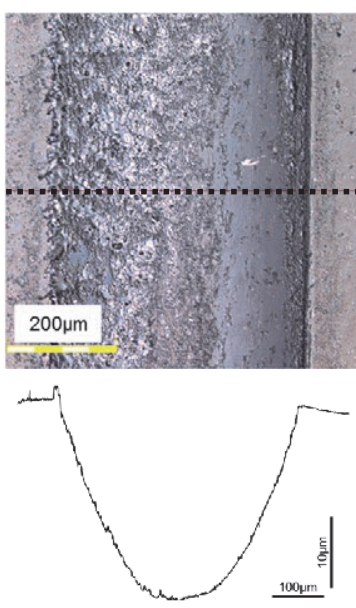

(c) 3.0 mass $\%$ oWNC/EP

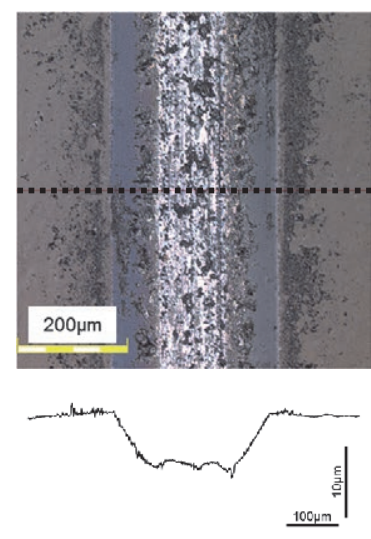

(b) 1.0 mass \% oWNC/EP
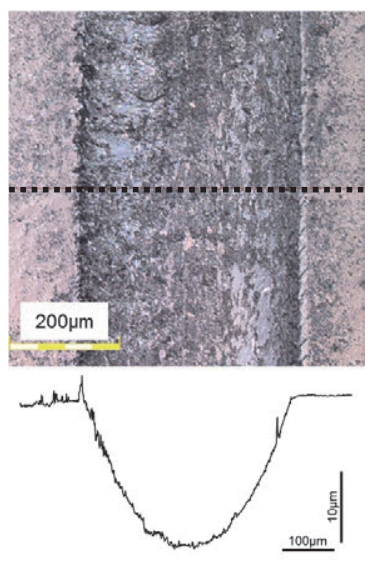

(d) 5.0 mass \% oWNC/EP
Fig. 13 Optical microscope images of the sliding surface of (a) 0.5 mass \%, (b) 1.0 mass\%, (c) 3.0 mass\%, and (d) 5.0 mass $\%$ oWNC/EP under water lubrication

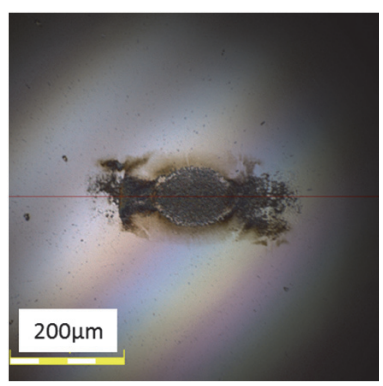

(a) Neat EP

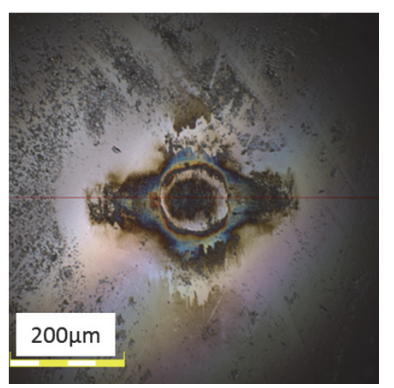

(b) 1.0 mass \% GO/EP

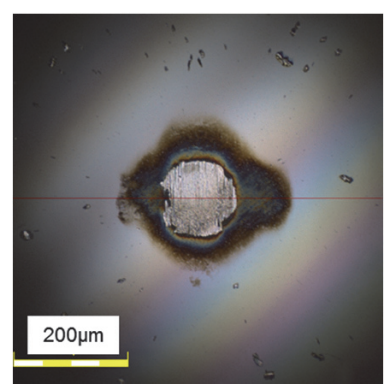

(c) 0.5 mass $\%$ oWNC/EP

Fig. 14 Optical microscope images of the wear scar of SUJ2 ball after the sliding with (a) neat epoxy, (b) 1.0 mass $\% \mathrm{GO} / \mathrm{EP}$, and (c) $0.5 \mathrm{mass} \%$ oWNC/EP under water lubrication 


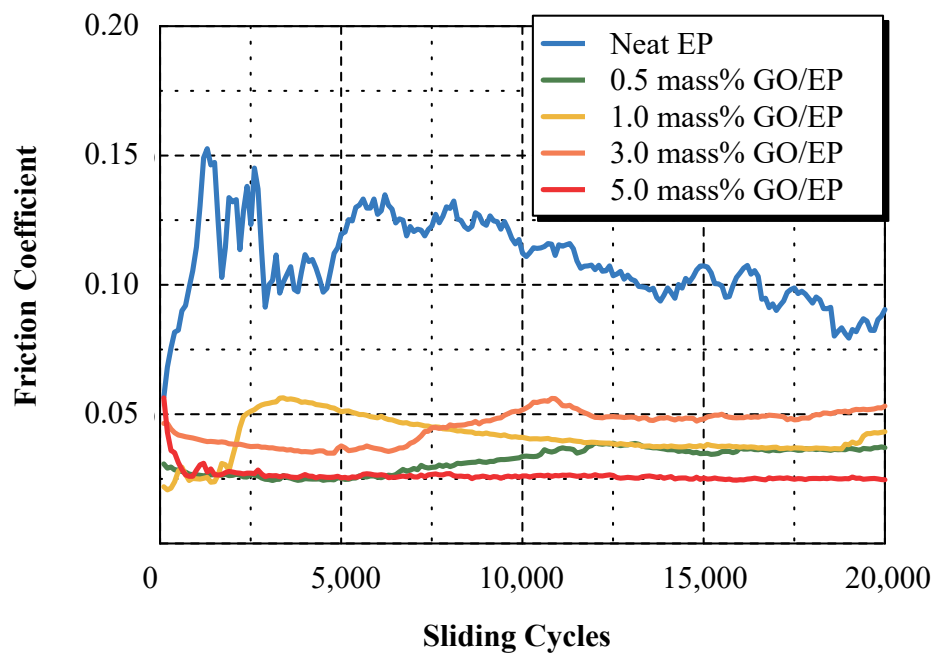

Fig. 15 Friction coefficients of 0-5.0 mass\% GO/EP under oil lubrication condition

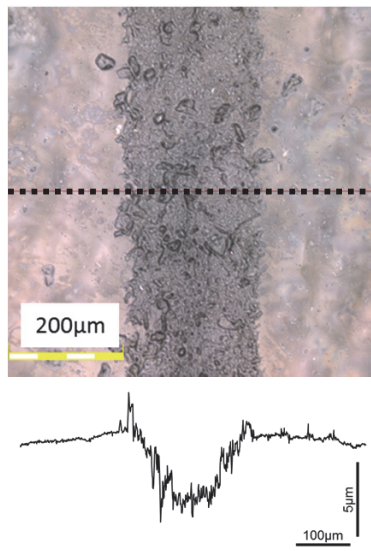

(a) Neat EP
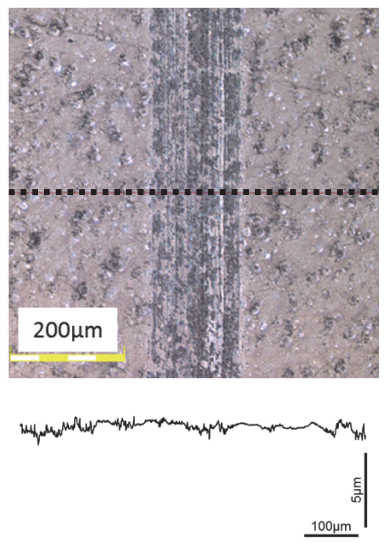

(d) 3.0 mass $\%$ GO/EP
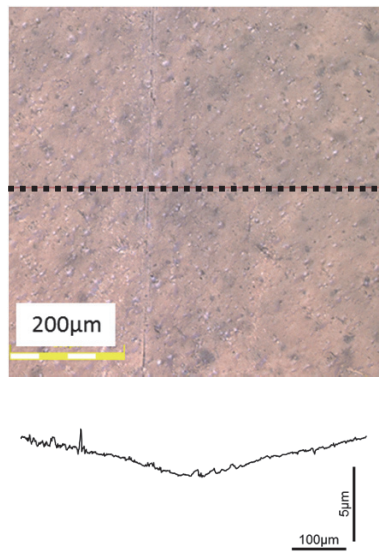

(b) 0.5 mass \% GO/EP
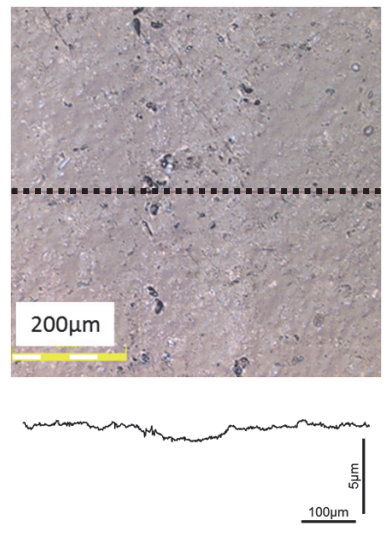

(e) 5.0 mass $\%$ GO/EP
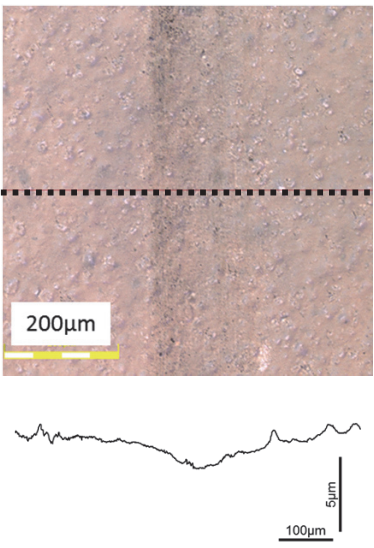

(c) 1.0 mass \% GO/EP

Fig. 16 Optical microscope images of the sliding surface of (a) neat epoxy, (b) 0.5 mass\%, (c) 1.0 mass $\%$, (d) 3.0 mass $\%$, and (e) 5.0 mass $\%$ GO/EP under oil lubrication 


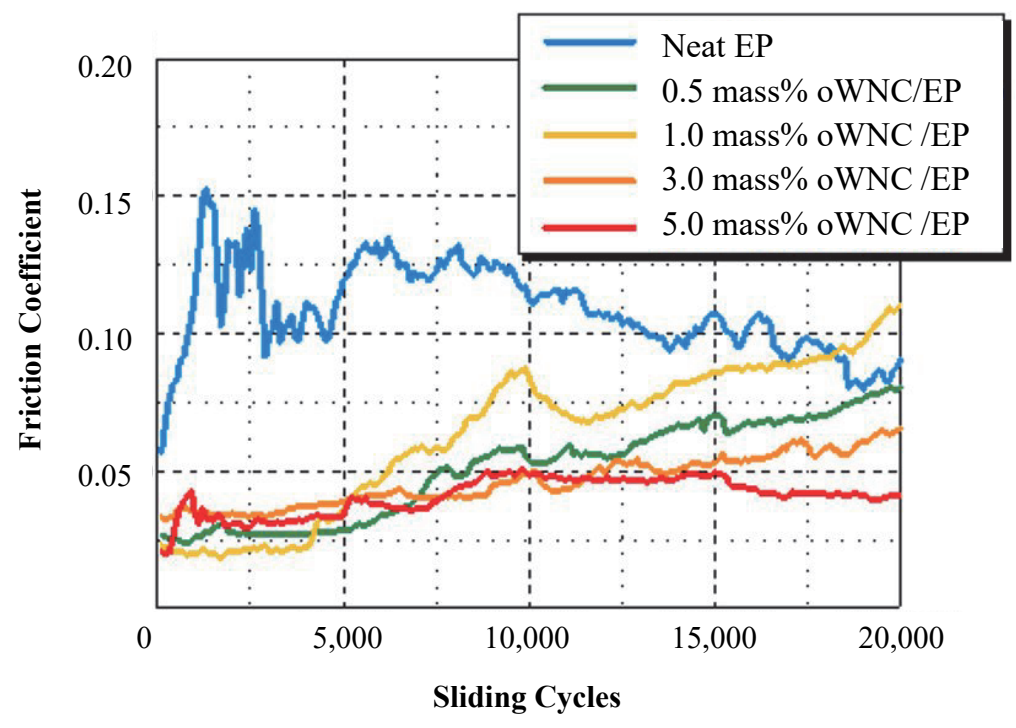

Fig. 17 Friction coefficients of 0-5.0 mass $\%$ oWNC/EP under oil lubrication condition

condition. Figure 18 shows the optical microscope images of the sliding surfaces of oWNC/EP under oil lubrication. Almost no wear was observed for all the specimens.

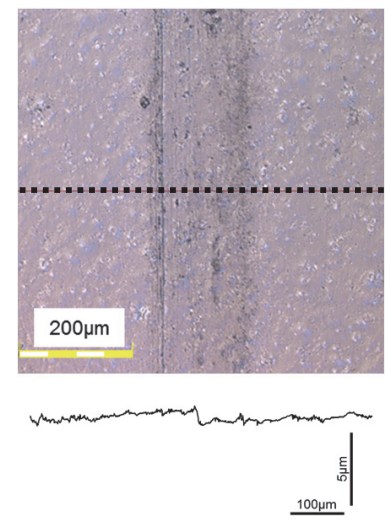

(a) 0.5 mass $\%$ oWNC/EP

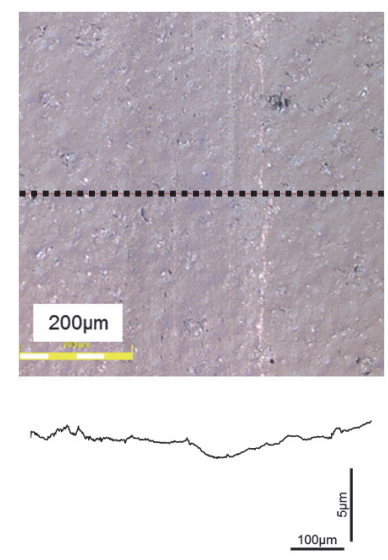

(c) 3.0 mass \% oWNC/EP

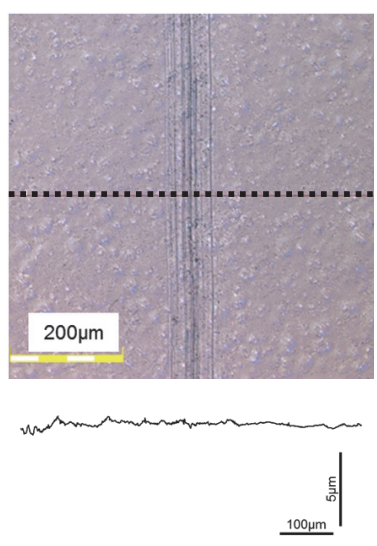

(b) 1.0 mass $\%$ oWNC/EP
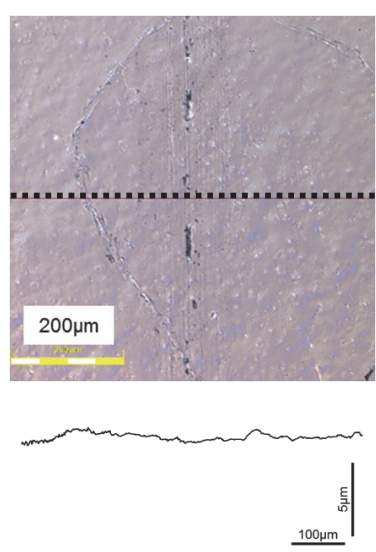

(d) 5.0 mass \% oWNC/EP
Fig. 18 Optical microscope images of the sliding surface of (a) 0.5 mass\%, (b) 1.0 mass\%, (c) 3.0 mass \%, and (d) 5.0 mass $\%$ oWNC/EP under oil lubrication

\section{Discussion}

Figure 19 summarizes the effect on the friction coefficients of GO/EP and oWNC/EP with additive concentration under dry, water, and oil lubrication conditions. The averaged friction coefficients after 10,000 sliding cycles were plotted. It was observed that the friction coefficients were inversely proportional to the additive concentration as they decreased with an increase in the additive concentration. A characteristic transition was observed for oWNC/EP under the water lubrication, where the friction coefficient rapidly decreased at a low concentration of 0.5 mass $\%$ and increased above 3.0 mass $\%$.

Figure 20 shows the effect on the wear of GO/EP and oWNC/EP plates with additive concentration under dry, water, and oil lubrication conditions. Wear resistance improvement of epoxy resin responsive to nanocarbons addition can be explained mainly by the increased hardness. The indentation hardness was monotonically increased at a higher additive concentration of both GO and oWNC. The increase of the hardness was due to the high elastic modulus of the nanocarbon materials. Lee et al. reported that the Young's modulus of a single-layer graphene is estimated to be $2.4 \mathrm{TPa}$ [25]. Under water lubrication conditions, the wear was higher than that under dry lubrication conditions. This effect may be explained by the softening of the epoxy under the water environment. The absorption of water leads to a decrease in the Young's modulus of the epoxy resin [26]. Nanocarbon materials were independent from water softening. This is thought to be one of the reasons for the high improving effect of nanocarbons on wear resistant under water lubrication condition.

Comparing the anti-wear properties of GO/EP and oWNC/ $\mathrm{EP}$, the lower concentration of oWNC/EP can effectively improve the anti-wear property of epoxy resin under water lubrication. The transition of the wear under water lubrication with the additive concentration was similar to that of the friction coefficient. This, therefore, implies that the wear properties of the composite materials affect the lubrication properties. Due to the two-dimensional configuration of GO, the orientation of GO in epoxy matrix may affect the lubrication properties. In general, graphitic structure gives low friction at the direction parallel to the layer. In contrast, the spherical configuration of 


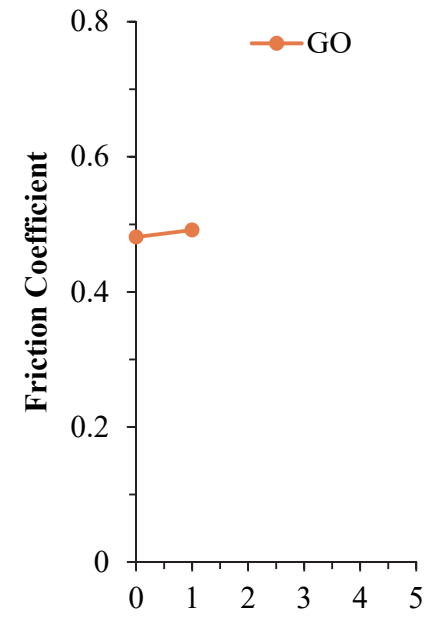

(a) Dry
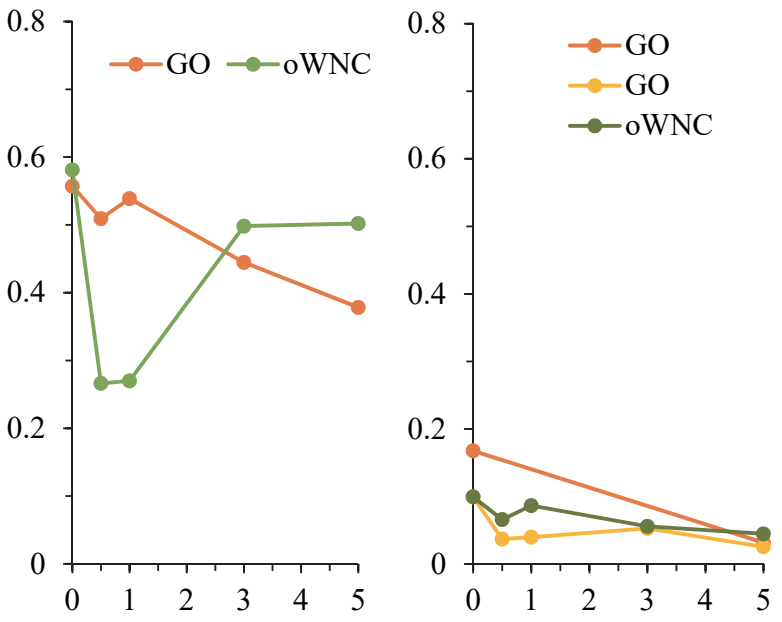

Additive Concentration (mass\%)

(b) Water lubrication

Fig. 19 Effect on the friction coefficients of GO/EP and oWNC/EP with additive concentration under (a) dry, (b) water, and (c) oil lubrication conditions

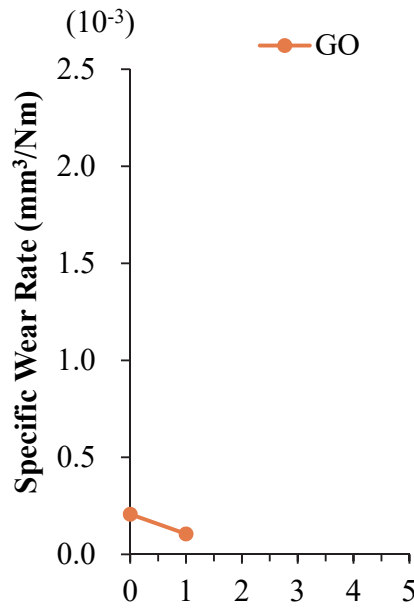

(a) Dry
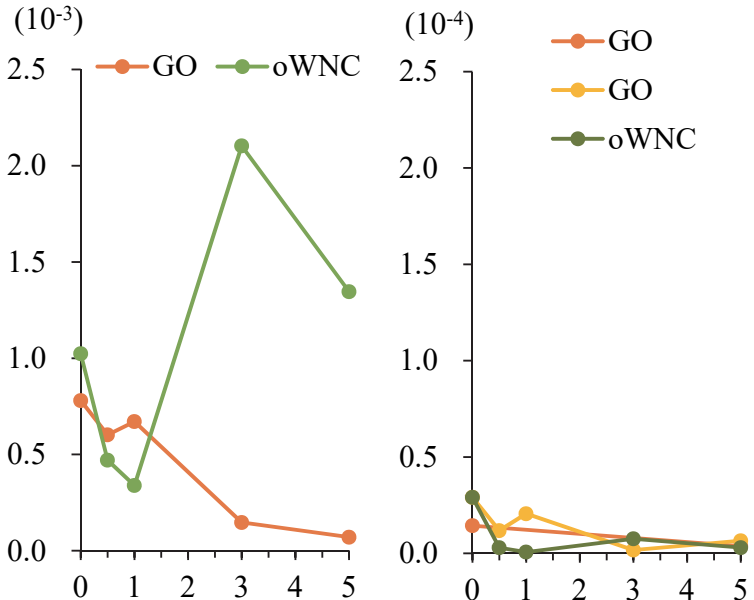

Additive Concentration (mass\%)

(b) Water lubrication

Fig. 20 Effect on the specific wear rate of GO/EP and oWNC/EP with additive concentration under (a) dry, (b) water, and (c) oil lubrication conditions

the oWNCs can allow them to show isotropic properties in the epoxy matrix. This configuration can lead to the low friction and low wear at a relatively low concentration of oWNC. However, anti-wear property of oWNC under water lubrication was degraded at a relatively high concentration of more than 3.0 mass $\%$. These effects were also observed when nanoparticles were added to the epoxy resin at a high concentration $[2,6]$. It is likely that the nanoparticles tend to agglomerate to form large particles at a high concentration in epoxy matrix. This might lead to an increase in the abrasive wear of the sliding surface. Therefore, additional improvement of wear resistant may be achieved by improving the agglomeration of oWNC.

\section{Conclusion}

The effect of the addition of GO and oWNC in epoxy resin on the mechanical and tribological properties under dry, water, and oil lubrication conditions was investigated. For mechanical properties such as tensile strength, tensile modulus, and indentation hardness, both nanomaterials improved the properties, but portrayed varying characteristics for tribological properties. It was revealed that the addition of $\mathrm{GO}$ and oWNC reduced the friction coefficient and wear of epoxy resin. Comparing the effect of GO and oWNC, a lower concentration of oWNC effectively improved the friction coefficient and 
wear under the water lubrication condition. However, a higher concentration of oWNC more than 3.0 mass\% in epoxy led to the degradation of anti-wear properties of the composite. The improvement of anti-wear properties of the epoxy resin was attributable to the increased hardness and lubricity with the addition of GO and oWNC. The isotropic spherical structure of oWNC might cause a higher effect on the lower additive concentration.

\section{Acknowledgments}

This research was partly supported by grants from Grantsin-Aid for Scientific Research (B) (17H03165, 26289028). The authors would like to thank Prof. Nishina of Okayama University for the supply of the GO.

\section{References}

[1] Shi, G., Zhang, M. Q., Rong, M. Z., Wetzel, B. and Friedrich, K., "Friction and Wear of Low Nanometer $\mathrm{Si}_{3} \mathrm{~N}_{4}$ Filled Epoxy Composites," Wear, 254, 7-8, 2003, 784-796.

[2] Xing, X. S. and Li, R. K. Y., "Wear Behavior of Epoxy Matrix Composites Filled with Uniform Sized Sub-Micron Spherical Silica Particles," Wear, 256, 1-2, 2004, 21-26.

[3] Ji, Q. L., Zhang, M. Q., Rong, M. Z., Wetzel, B. and Friedrich, K., "Friction and Wear of Epoxy Composites Containing Surface Modified SiC Nanoparticles," Tribology Letters, 20, 2, 2005, 115-123.

[4] Zhang, Z., Breidt, C., Chang, L., Haupert, F. and Friedrich, K., "Enhancement of the Wear Resistance of Epoxy: Short Carbon Fibre, Graphite, PTFE and Nano-TiO ${ }_{2}$ " Composites Part A: Applied Science and Manufacturing, 35, 12, 2004, 1385-1392.

[5] Chang, L., Zhang, Z., Ye, L. and Friedrich, K., “Tribological Properties of Epoxy Nanocomposites," Wear, 262, 5-6, 2007, 699706.

[6] Lin, J.-C., "Compression and Wear Behavior of Composites Filled with Various Nanoparticles," Composites Part B: Engineering, 38, 1, 2007, 79-85.

[7] Jacobs, O., Xu, W., Schädel, B. and Wu, W., "Wear Behaviour of Carbon Nanotube Reinforced Epoxy Resin Composites," Tribology Letters, 23, 1, 2006, 65-75.

[8] Zhang, L. C., Zarudi, I. and Xiao, K. Q., "Novel Behaviour of Friction and Wear of Epoxy Composites Reinforced by Carbon Nanotubes," Wear, 261, 7-8, 2006, 806-811.

[9] Zhao, F., Zhang, L., Li, G., Guo, Y., Qi, H. and Zhang, G., "Significantly Enhancing Tribological Performance of Epoxy by Filling with Ionic Liquid Functionalized Graphene Oxide," Carbon, 136, 2018, 309-319.

[10] Upadhyay, R. K. and Kumar, A., "Effect of Humidity on the Synergy of Friction and Wear Properties in Ternary Epoxy-Graphene-MoS Composites," Carbon, 146, 2019, 717-727.

[11] Zhou, H., Wang, H., Du, X., Mo, Y., Yuan, H. and Liu, H.-Y., “Hybrid Three-Dimensional Graphene Fillers and Graphite Platelets to Improve the Thermal Conductivity and Wear Performance of Epoxy Composites," Composites Part A: Applied Science and Manufacturing, 123, 2019, 270-277.

[12] Yang, H., Shan, C., Li, F., Zhang, Q., Han, D. and Niu, L., "Convenient Preparation of Tunably Loaded Chemically Converted
Graphene Oxide/Epoxy Resin Nanocomposites from Graphene Oxide Sheets through Two-Phase Extraction," Journal of Materials Chemistry, 19, 46, 2009, 8732-8740.

[13] Miller, S. G., Bauer, J. L., Maryanski, M. J., Heimann, P. J., Barlow, J. P., Gosau, J.-M. and Allred, R. E., "Characterization of Epoxy Functionalized Graphite Nanoparticles and the Physical Properties of Epoxy Matrix Nanocomposites," Composites Science and Technology, 70, 7, 2010, 1120-1125.

[14] Neitzel, I., Mochalin, V., Bares, J. A., Carpick, R. W., Erdemir, A. and Gogotsi, Y., "Tribological Properties of Nanodiamond-Epoxy Composites," Tribology Letters, 47, 2, 2012, 195-202.

[15] Cui, L.-J., Geng, H.-Z., Wang, W.-Y., Chen, L.-T. and Gao, J., "Functionalization of Multi-Wall Carbon Nanotubes to Reduce the Coefficient of the Friction and Improve the Wear Resistance of Multi-Wall Carbon Nanotube/Epoxy Composites," Carbon, 54, 2013, $277-282$.

[16] Shen, X.-J., Pei, X.-Q., Fu, S.-Y. and Friedrich, K., “Significantly Modified Tribological Performance of Epoxy Nanocomposites at Very Low Graphene Oxide Content," Polymer, 54, 3, 2013, 12341242.

[17] Shen, X.-J., Pei, X.-Q., Liu, Y. and Fu, S.-Y., "Tribological Performance of Carbon Nanotube-Graphene Oxide Hybrid/Epoxy Composites," Composites Part B: Engineering, 57, 2014, 120-125.

[18] Ribeiro, H., da Silva, W. M., Neves, J. C., Calado, H. D. R., Paniago, R., Seara, L. M., das Mercês Camarano, D. and Silva, G. G., "Multifunctional Nanocomposites Based on Tetraethylenepentamine-Modified Graphene Oxide/Epoxy," Polymer Testing, 43, 2015, 182-192.

[19] Matsuura, K., Umahara, Y., Gotoh, K., Hoshijima, Y. and Ishida, H., "Surface Modification Effects on the Tensile Properties of Functionalised Graphene Oxide Epoxy Films," RSC Advances, 8, 18, 2018, 9677-9684.

[20] Potts, J. R., Dreyer, D. R., Bielawski, C. W. and Ruoff, R. S., “Graphene-Based Polymer Nanocomposites," Polymer, 52, 1, 2011, $5-25$.

[21] Kinoshita, H. and Nishina, Y., "Investigations on Tribological Mechanisms of Graphene Oxide and Oxidized Wood-Derived Nanocarbons as Water-Based Lubricating Additives," Tribology Online, 11, 2, 2016, 235-241.

[22] Kodama, Y., Sato, K., Suzuki, K., Saito, Y., Suzuki, T. and Konno, T. J., "Electron Microscope Study of the Formation of Graphitic Nanostructures in Nickel-Loaded Wood Char," Carbon, 50, 10, 2012, 3486-3496.

[23] Dreyer, D. R., Park, S., Bielawski, C. W. and Ruoff, R. S., "The Chemistry of Graphene Oxide," Chem. Soc. Rev., 39, 1, 2010, 228 240.

[24] Sevilla, M., Sanchís, C., Valdés-Solís, T., Morallón, E. and Fuertes, A. B., "Direct Synthesis of Graphitic Carbon Nanostructures from Saccharides and Their Use as Electrocatalytic Supports," Carbon, 46, 6, 2008, 931-939.

[25] Lee, J.-U., Yoon, D. and Cheong, H., "Estimation of Young's Modulus of Graphene by Raman Spectroscopy," Nano Letters, 12, 9, 2012, 4444-4448.

[26] De'Nève, B. and Shanahan, M. E. R., "Water Absorption by an Epoxy Resin and Its Effect on the Mechanical Properties and InfraRed Spectra," Polymer, 34, 24, 1993, 5099-5105. 